\title{
Augmented Two-channel Arrhythmia Detection
}

\author{
Chih-ming James Chiang, MS, Janice M. Jenkins, PhD, and \\ Lorenzo A. DiCarlo, MD
}

It has been estimated that up to $40 \%$ of all electrical shocks delivered by implantable cardioverter-defibrillators (ICDs) result from false positive detection of ventricular arrhythmias. To prevent false diagnosis of such tachyarrhythmias in ICDs, detection methods complementary to rate analysis must bc combincd in an algorithm suitable for implementation in next generation ICDs.

\section{Materials and Methods}

The augmented two-channel arrhythmia detection (A2CAD) method, an on-line algorithm that employs information from both the atrial and ventricular channels, has been designed for arrhythmia diagnosis for implantable devices. The scheme utilizes rate analysis of atrial and ventricular activation as a preliminary analysis. ${ }^{1}$ This is sufficient to recognize most arrhythmias in which atrial activation predominates or in cases in which ventricular activation predominates. In cases where atrial and ventricular activation are equal, that is, a $1: 1$ relationship, morphological analysis of each channel is invoked to further characterize the arrhythmia. ${ }^{2}$ Morphology is classified as normal or abnormal by computing a cross correlation between the waveform under analysis and a stored template.

From the Departments of Electrical Engineering and Computer Science Medicine, The Iniversity of Michigan, Ann Arbor, Michigan.

Supported in part by Medtronic, Inc., and the National Science Foundation Grant EID-9023514.

Reprint requests: Chih-ming Jamcs Chiang, MS, Mcdical Computing Laboratory, University of Michigan, 1301 Beal Street, Ann Arbor, MI 48109-2122.

\section{Results}

A2CAD was tested on recordings of bipolar intraatrial and intraventricular electrograms $(1 \mathrm{~cm}$, $1-500 \mathrm{~Hz}$ ) acquired during electrophysiology studies. The 40 patient cases ( $10-50 \mathrm{~s}$ ) analyzed included 6 atrial flutters, 4 atrial fibrillations, 6 supraventricular tachycardias, 2 sinus tachycardias, 12 ventricular tachycardias, 7 ventricular flutters, and 3 ventricular fibrillations. A2CAD successfully diagnosed 40 of 40 cases for an overall success rate of $100 \%$.

\section{Conclusion}

An innovative method (A2CAD), which uses rate augmented by morphologic analysis of both atrial and ventricular channels to derive accurate diagnosis of complex arrhythmias, has been shown to be effective and efficient. Incorporation of fast morphological algorithms ${ }^{3}$ is planncd for future versions, which may be feasible for use in implantable devices.

\section{References}

1. Arzbaecher R, Bump T, Jenkins J: Automatic tachycardia recognition. PACE 7:541, 1984

2. Lin D, DiCarlo L, Jenkins J: Identification of ventricular tachycardia using intracavitary ventricular electrograms: analysis of time and frequency domain patterns. PACE 11:1592, 1988

3. Throne R, Jenkins J, DiCarlo L: A comparison of four new time-domain techniques for discriminating monomorphic ventricualr tachycardia from sinus rhythm using ventricular waveform morphology. IEEE Trans Biomed Eng 38:561, 1991 